\title{
The Association between Evidence-Based Healthcare Practices and Outcomes among Preterm Births in China
}

\author{
Di Jin ${ }^{a} \quad$ Xinyue Gu ${ }^{b}$ Siyuan Jiang ${ }^{c}$ Yanchen Wang ${ }^{b} \quad$ Tongling Yang $^{c}$ \\ Yulan Lu ${ }^{d}$ Yun Caoc Shoo K. Lee ${ }^{e, f, g}$ Hui Wu$^{a}$ on behalf of the CHNN \\ investigators
}

\begin{abstract}
${ }^{a}$ Department of Neonatology, The First Hospital of Jilin University, Changchun, China; ${ }^{\mathrm{b}} \mathrm{NHC}$ Key Laboratory of Neonatal Diseases (Fudan University), Children's Hospital of Fudan University, Shanghai, China; 'Department of Neonatology, Children's Hospital of Fudan University, Shanghai, China; ${ }^{\mathrm{d} C e n t e r}$ for Molecular Medicine, Pediatrics Research Institute, Children's Hospital of Fudan University, Shanghai, China; 'Maternal-Infant Care Research Centre, Department of Pediatrics, Mount Sinai Hospital, Toronto, ON, Canada; fDepartment of Pediatrics, University of Toronto, Toronto, ON, Canada; 9 Department of Obstetrics and Gynecology, Dalla Lana School of Public Health, University of Toronto, Toronto, ON, Canada
\end{abstract}

\section{Keywords}

Preterm · Evidence-based healthcare practices · Death ·

Severe brain injury

\begin{abstract}
Introduction: Very preterm infants are at high risk of early death or severe brain injury, with potential for impaired long-term neurodevelopmental function and physical health. There are evidence-based healthcare practices that can reduce the incidence. Materials and Methods: Infants born at $24-31^{6}$ weeks gestational age and admitted within $24 \mathrm{~h}$ to NICUs participating in the Chinese Neonatal Network in 2019 were included. We examined the association between 4 evidence-based practices: inborn (born in a tertiary hospital in the Chinese Neonatal Network), ACS (any antenatal corticosteroid), $\mathrm{MgSO}_{4}$ (prenatal magnesium sulfate), and NT (normothermic temperature $\left[36.0-37.5^{\circ} \mathrm{C}\right]$ at admission) and early death and/or severe brain injury in the study pop-
\end{abstract}

karger@karger.com www.karger.com/neo

Karger $\stackrel{\text { ' }}{5}$

BOPEN ACCESS
(C) 2021 The Author(s)

Published by S. Karger AG, Basel

This is an Open Access article licensed under the Creative Commons Attribution-NonCommercial-4.0 International License (CC BY-NC) (http://www.karger.com/Services/OpenAccessLicense), applicable to the online version of the article only. Usage and distribution for commercial purposes requires written permission. ulation. Results: Of 6,035 eligible infants, the incidence of early death and/or severe brain injury was $10.6 \%$. Exposure to ACS only was associated with significant lower incidence of death and/or severe brain injury than none (aOR, 0.71; $95 \% \mathrm{Cl}: 0.57-0.88)$, but not $\mathrm{MgSO}_{4}$ only (aOR, $0.97 ; 95 \% \mathrm{Cl}$ : $0.81-1.17)$, NT only (aOR, $0.91 ; 95 \% \mathrm{Cl}: 0.76-1.08$ ), or inborn only (aOR, $0.91 ; 95 \% \mathrm{Cl}: 0.72-1.15)$. The association between number of practices and incidence of early death and/or severe brain injury is as follows: none $=23 \%(31 / 138)$, any $1=$ $14 \%(84 / 592)$, any $2=12 \%(185 / 1,538)$, any $3=9 \%(202 / 2,285)$, and all $4=9 \%(140 / 1,482)$. Discussion/Conclusion: More comprehensive use of evidence-based practices was associated with improved survival without severe brain injury among very preterm infants born at $<32$ weeks gestational age.

(C) 2021 The Author(s)

Published by S. Karger AG, Basel

A complete list of group members appears in the Acknowledgment section.
Correspondence to:

Hui Wu,wuhui@jlu.edu.cn 


\section{Introduction}

Preterm birth is the leading cause of neonatal mortality and morbidity in the world. According to the WHO, China has a preterm birth rate of $6.9 \%$ and the second highest absolute number of preterm births (1.16 million per annum) in the world [1]. Although very preterm infants (VPI) born at $<32$ weeks gestational age (GA) represent only $2 \%$ of all live births or $16 \%$ of all preterm births, they have the highest mortality and morbidity, including adverse long-term neurodevelopmental outcomes [2].

During the past decade, several studies have reported that select maternal and newborn practices can improve preterm birth outcomes [3], including delivery in tertiary centers $[4,5]$, administration of antenatal corticosteroids $[6,7]$, prenatal $\mathrm{MgSO}_{4}$ usage $[8,9]$, and maintenance of normal body temperature [10]. These 4 practices have been shown to be effective in clinical trials and are widely used clinically. However, the efficacy of clinical interventions in the real world is often different from randomized controlled trials since population risks may differ and multiple interventions may coexist. Our objective was to examine the individual and cumulative effects of these 4 evidence-based clinical practices in the VPI population in China and on their subpopulation of extremely preterm infants (EPI) born at $24-27^{6}$ weeks GA.

\section{Methods}

\section{The Chinese Neonatal Network and Database}

The Chinese Neonatal Network (CHNN) is a group of multidisciplinary researchers from 57 tertiary-level NICUs in 25 provinces and municipalities that are representative of different geographic regions of China. In 2019, 57 NICUs contributed to the CHNN, which prospectively collects standardized data on demographics, outcomes, selected clinical practices, and resource use on all VPI born at $24^{0}-31^{6}$ weeks GA or with a birth weight $<1,500 \mathrm{~g}$ and admitted to participating hospitals. This project was approved by the CHNN Executive Committee and the Research Ethics Board at the Children's Hospital of Fudan University, Shanghai, China [11].

\section{Study Population}

The population for this study consisted of VPI born at GA of $24^{0}-31^{6}$ weeks and admitted to participating CHNN NICUs within $24 \mathrm{~h}$ after birth from January 2019 to December 2019.

\section{Outcome}

The primary outcome was early death or severe brain injury. Early death was defined as death within 7 days of age before discharge from the NICU. Severe brain injury was defined as intraventricular hemorrhage grade III or IV according to Papile's classification $[2,12]$.

Association between Practices and

Outcomes among Preterm Births
Exposure Variables

We used 4 evidence-based practices as exposure variables since they are identified by the literature to be associated with the primary outcome: (1) inborn status, defined as born in a tertiary hospital that participates in the CHNN; (2) ACS, defined as exposure to any antenatal corticosteroids; (3) $\mathrm{MgSO}_{4}$, defined as administration of magnesium sulfate before delivery for any reason; (4) NT, defined as normal body temperature on NICU admission between 36.0 and $37.5^{\circ} \mathrm{C}$.

\section{Statistical Analyses}

Descriptive statistical methods were used to summarize the study population. The infant and maternal characteristics were compared between 2 groups: infants with or without the composite of early death or severe brain injury (primary outcome) using the $\chi^{2}$ test for categorical variables and Student $t$ test or Wilcoxon rank-sum test, as appropriate, for continuous variables. To investigate the association between the 4 clinical practices (exposures) and the primary outcome, we used the $\chi^{2}$ test to compare the 4 practices, individually and cumulatively. We used multivariate logistic regression models to adjust for potential confounders that were identified in the univariate analyses or based on clinical experience, including gestational age, small for gestational age, sex, Apgar score $<7$ at $5 \mathrm{~min}$, delivery mode, and gestational diabetes. The Generalized Estimating Equation (GEE) approach was used to account for the clustering of infants within NICUs.

To determine the most effective combination group of these 4 practices, we examined the adjusted OR (aOR) of the outcome with different combinations of practices versus no practices using similar models mentioned above. We examined the effect of 2, 3, and 4 practices on the outcome by estimating the percent change in the rate of primary outcome from the observed rate of outcome to the predicted rate of outcome given the exposure of at least 2, 3, and 4 practices based on the multiple logistic regression model adjusted for the potential confounders mentioned above. We also conducted subgroup analyses stratified by GA groups (e.g., $24-27^{6}$ weeks and $28-31^{6}$ weeks) using similar methods.

The data management and all statistical analyses were performed using SAS version 9.4 (SAS Institute, Inc., Cary, NC, USA). Two-sided $p$ value of 0.05 was used to determine statistical significance.

\section{Results}

During the study period, 10,823 infants were admitted to the 57 CHNN NICUs. Among them, 8,016 infants with GA between 24 and $31^{6}$ weeks were admitted to the NICUs in CHNN within $24 \mathrm{~h}$ in 2019. We excluded 56 infants with major congenital anomalies according to the CHNN data definition manual, 960 infants with missing information on the 4 practices, and 965 infants with missing outcome. The remaining 6,035 eligible infants were included for analyses (Fig. 1).

The incidence of the primary outcome, early death or severe brain injury, was $10.6 \%$ in the study cohort. The in- 
Table 1. Incidence of early death and/or severe brain injury among preterm infants

\begin{tabular}{|c|c|c|c|c|}
\hline Gestational age, weeks & $N$ & $\begin{array}{l}\text { Early death, } \\
n / N(\%)\end{array}$ & $\begin{array}{l}\text { Severe brain injury, } \\
n / N(\%)^{a}\end{array}$ & $\begin{array}{l}\text { Early death and/or severe } \\
\text { brain injury, } n / N(\%)\end{array}$ \\
\hline $24^{+0}-27^{+6}$ & 911 & 80/911 (8.8) & $157 / 870(18.1)$ & $212 / 911(23.3)$ \\
\hline $28^{+0}-31^{+6}$ & 5,124 & $91 / 5,124(1.8)$ & $359 / 5,079(7.1)$ & $430 / 5,124(8.4)$ \\
\hline All & 6,035 & $171 / 6,035$ (2.8) & $516 / 5,949(8.7)$ & $642 / 6,035$ (10.6) \\
\hline
\end{tabular}

a 86/6,035 infants missing neuroimaging results due to early death before they would have been screened.

Table 2. Proportion of infants for each individual healthcare practice

\begin{tabular}{llllll}
\hline Gestational age, weeks & $N$ & Inborn, $n(\%)$ & ACS, $n(\%)$ & MgSO $_{4}, n(\%)$ & NT, $n(\%)$ \\
\hline $24^{+0}-27^{+6}$ & 911 & $770(84.5)$ & $695(76.3)$ & $456(50.1)$ & $458(50.3)$ \\
$28^{+0}-31^{+6}$ & 5,124 & $4,269(83.3)$ & $4,145(80.9)$ & $2,638(51.5)$ & $3,020(58.9)$ \\
\hline All & 6,035 & $5,039(83.5)$ & $4,840(80.2)$ & $3,094(51.3)$ & $3,478(57.6)$
\end{tabular}

Four Evidence-Based Practices: inborn, born in a tertiary hospital within CHNN; ACS, administration of antenatal corticosteroids for any course; $\mathrm{MgSO}_{4}$, application of magnesium sulfate before delivery for any reason; NT, maintenance of normal body temperature on admission within $\left(36.0-37.5^{\circ} \mathrm{C}\right)$. ACS, antenatal corticosteroids; NT, normothermia.

Fig. 1. Flowchart for study population. GA, gestational age.

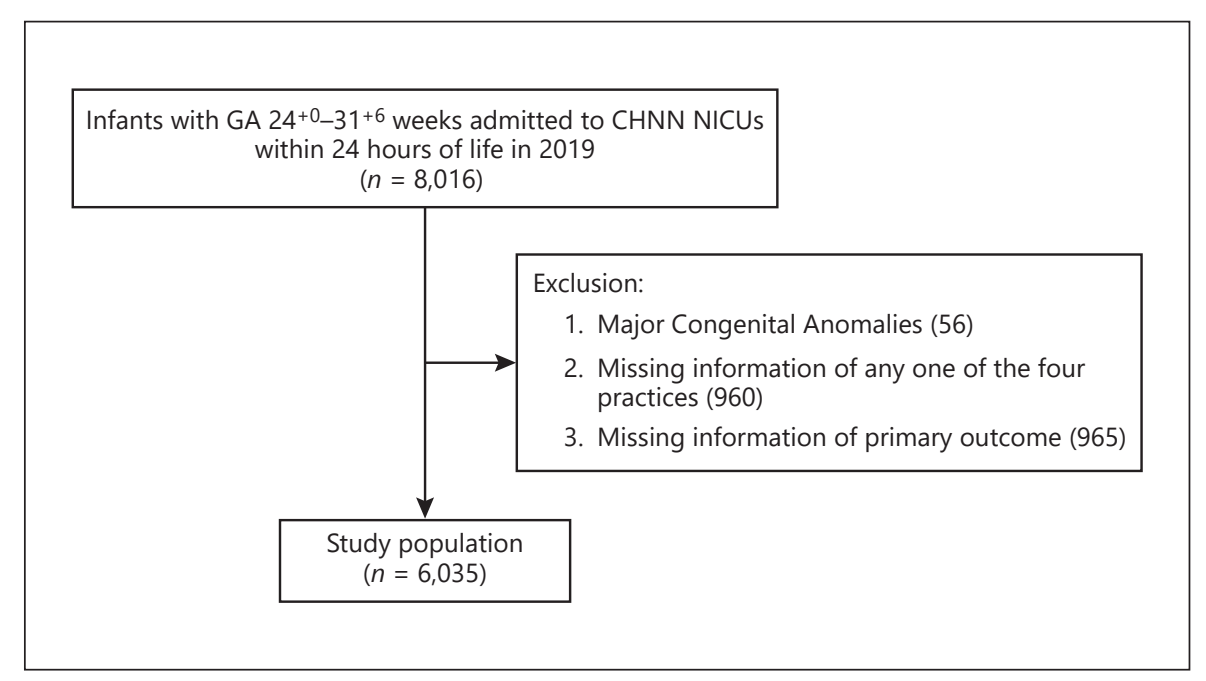

cidence was much higher among infants born at $24-27^{6}$ weeks GA compared to those at $28-31^{6}$ weeks GA $(23.3 \%$ vs. $8.4 \%$, respectively) (Table 1 ). Among the 4 evidencebased practices, the incidence of exposure to inborn was $83.5 \%(n=5,039)$, ACS was $80.2 \%(n=4,840), \mathrm{MgSO}_{4}$ was $51.3 \%(n=3,094)$, and NT was $57.6 \%(n=3,478)$ (Table 2$)$.

Comparisons of maternal and infant characteristics are shown in Table 3. Infants who survived without severe brain injury were significantly $(p<0.05)$ more likely to have maternal diabetes and be born by caesarean section than those who died or had severe brain injury. They also had significantly $(p<0.05)$ higher median GA and mean birth weight and were less likely to be male and have Apgar score $<7$ at $5 \mathrm{~min}$.

Table 4 shows that exposure to any single practice was not significantly associated with lower incidence of early 
Table 3. Characteristics of infants who survived without severe brain injury compared with those who died or developed severe brain injury

\begin{tabular}{|c|c|c|c|}
\hline Characteristics, $n / N(\%)^{a}$ & $\begin{array}{l}\text { Survival without severe } \\
\text { brain injury }(N=5,393)\end{array}$ & $\begin{array}{l}\text { Early death and/or } \\
\text { severe brain injury }(N=642)\end{array}$ & $p$ value \\
\hline \multicolumn{4}{|l|}{ Maternal characteristics } \\
\hline Maternal age, mean (SD), years & $31.0(4.8)$ & $31.1(4.9)$ & 0.49 \\
\hline Maternal diabetes & $989 / 5,384(18.4)$ & $80 / 639(12.5)$ & $<0.01$ \\
\hline Maternal hypertension & $1,021 / 5,386(19.0)$ & $126 / 641(19.7)$ & 0.67 \\
\hline Chorioamnionitis $^{b}$ & $622 / 4,136(15.0)$ & $83 / 461(18.0)$ & 0.09 \\
\hline $\mathrm{ROM}>24 \mathrm{~h}$ & $1,412 / 5,304(26.6)$ & $148 / 624(23.7)$ & 0.12 \\
\hline Cesarean section & $3,144 / 5,388(58.4)$ & $327 / 639(51.2)$ & $<0.01$ \\
\hline \multicolumn{4}{|l|}{ Births, $n$} \\
\hline Singleton & $3,782 / 5,393(70.1)$ & $431 / 642(67.1)$ & \\
\hline Twins & $1,531 / 5,393(28.4)$ & $199 / 642(31.0)$ & 0.26 \\
\hline Triplets or more & $80 / 5,393(1.5)$ & $12 / 642(1.9)$ & \\
\hline \multicolumn{4}{|l|}{ Infant characteristics } \\
\hline Birth weight, mean (SD), g & $1,358.1(308.4)$ & $1,198.9(332.2)$ & $<0.01$ \\
\hline Gestational age, median (IQR), weeks & $30.0(28.7,31.0)$ & $28.7(27.1,30.1)$ & $<0.01$ \\
\hline Small for $G A,<10$ th percentile & $354 / 5,393(6.6)$ & $41 / 642(6.4)$ & 0.86 \\
\hline Male & $3,012 / 5,390(55.9)$ & $390 / 642(60.7)$ & 0.02 \\
\hline Apgar score $<7$ at $5 \mathrm{~min}$ & $203 / 5,252(3.9)$ & $84 / 621(13.5)$ & $<0.01$ \\
\hline
\end{tabular}

GA, gestational age. ${ }^{a}$ All percentages are based on available data. ${ }^{b}$ Chorioamnionitis has $24 \%$ missing data, while all other variables have less than $3 \%$.

Table 4. The association between the individual practice and the primary outcome of early death or severe brain injury

\begin{tabular}{|c|c|c|c|c|c|c|}
\hline \multirow[t]{2}{*}{ Exposure } & \multicolumn{2}{|c|}{ Crude OR (95\% Cl) } & \multicolumn{4}{|c|}{ Adjusted OR $(95 \% \mathrm{Cl})^{a}$} \\
\hline & $24^{+0}-27^{+6}$ & $28^{+0}-31^{+6}$ & all & $24^{+0}-27^{+6}$ & $28^{+0}-31^{+6}$ & all \\
\hline \multicolumn{7}{|c|}{ Individual practice } \\
\hline Inborn & $0.76(0.51,1.14)$ & $0.84(0.65,1.08)$ & $0.83(0.68,1.03)$ & $0.89(0.55,1.44)$ & $0.90(0.68,1.18)$ & $0.91(0.72,1.15)$ \\
\hline ACS & $0.50(0.36,0.71)$ & $0.70(0.55,0.88)$ & $0.61(0.50,0.73)$ & $0.58(0.39,0.87)$ & $0.77(0.60,1.00)$ & $0.71(0.57,0.88)$ \\
\hline $\mathrm{MgSO}_{4}$ & $0.78(0.57,1.06)$ & $0.83(0.68,1.01)$ & $0.81(0.69,0.95)$ & $1.09(0.76,1.57)$ & $0.94(0.76,1.16)$ & $0.97(0.81,1.17)$ \\
\hline NT & $0.75(0.55,1.02)$ & $0.88(0.72,1.07)$ & $0.79(0.67,0.93)$ & $0.76(0.55,1.07)$ & $0.97(0.79,1.19)$ & $0.91(0.76,1.08)$ \\
\hline
\end{tabular}

ACS, antenatal corticosteroids; NT, normothermia. ${ }^{a}$ Adjusted for gestational age, small for gestational age, sex, Apgar score $<7$ at 5 min, delivery type, and gestational diabetes.

death or severe brain injury (aOR 0.64 [95\% CI: 0.38 , 1.08]). However, when individual specific practices were considered, ACS was associated with lower incidence of early death or severe brain injury (aOR 0.71 [95\% CI: 0.57, $0.88]$ ). Sensitivity analysis using the inversed propensity score weighting (IPSW) method for sensitivity analysis produced similar results (online suppl. Table 1; see www.karger.com/doi/10.1159/000519846 for all online suppl. material). Subgroup analyses were not significant for both the $24^{0}-27^{6}$ weeks GA and $28^{0}-31^{6}$ weeks GA subgroups. Inborn status, $\mathrm{MgSO}_{4}$, and NT were not indi- vidually associated with a significantly lower incidence of early death or severe brain injury. Sensitivity analysis excluding births at the lowest gestational ages (online suppl. Table 2) produced similar results.

Among the study cohort, 138 (2.3\%) infants received none of the 4 practices, $592(9.8 \%)$ infants received 1 practice, 1,538 (25.5\%) infants received 2 practices, 2,285 (37.9\%) infants received 3 practices, and 1,482 (24.5\%) infants received all 4 practices. Online supplementary Table 3 shows that exposure to 2, 3, or 4 practices was associated with significantly $(p<0.05)$ lower rate of death or 
severe brain injury - any 2 practices (aOR 0.55 [95\% CI: $0.34,0.90]$ ), any 3 practices (aOR 0.41 [95\% CI: 0.26 , $0.67]$ ), all 4 practices (aOR 0.50 [95\% CI: $0.30,0.81]$ ). The lowest incidence of early death or severe brain injury occurred with exposure to ACS + $\mathrm{MgSO}_{4}$ (aOR $0.38[95 \%$ CI: $0.18,0.81])$ and inborn + ACS + NT (aOR $0.39[95 \%$ CI: $0.23,0.65]$ ). Sensitivity analysis using the IPSW method produced similar results (online suppl. Table 4). Subgroup analysis showed that combinations of exposures that resulted in a significantly $(p<0.05)$ lower rate of early death or severe brain injury were only detected among infants $28^{0}-31^{6}$ weeks GA but not among infants $24^{0}-27^{6}$ weeks GA. Sensitivity analysis excluding births at the lowest gestational ages (online suppl. Table 5) produced similar results.

\section{Discussion}

In this large cohort of 6,035 VPI in China, the incidence of severe brain injury was $8.7 \%$, which is significantly lower than that reported by Kong et al. [13] in China 5 years ago, but still higher than that in other developed countries, such as $7.7 \%$ from the Canadian Neonatal Network in 2019 [14] and 6.2\% from the EPICE population-based cohort in 2011 [15]. This suggests that although outcomes in Chinese NICUs have improved, there is still room for improvement, and quality-improvement efforts using evidence-based practices may further improve neurodevelopmental and other outcomes.

To our knowledge, this is the first large multicenter study of the association between 4 evidence-based practices and the incidence of early death or severe brain injury among VPI in China. Of the 4 practices examined in this study, the incidence of inborn was the highest, at $83.5 \%$. This compares favorably with reports from developed countries like Canada (73.5\% for infants on CNN in 2019) [14] and Australia-New Zealand (88.1\% for infants $<32$ weeks GA in 2017) [16]. There is ample evidence that preterm infants born at tertiary institutions (inborn) where the appropriate level of neonatal care is available have better outcomes, including early death and severe brain injury, than those born elsewhere and then require transportation to tertiary centers for care $[4,5]$. While we did not find an association between inborn and early death or severe brain injury, this may be because many of the hospitals in the CHNN are major regional centers with well-developed regionalization and patient referral and transport systems.
The prevalence of exposure to ACS in our cohort was $80.2 \%$, which is significantly increased over the $56 \%$ incidence reported by Kong et al. [13] in China in 2013-2014. ACS exposure was lower among infants born at $24-27^{6}$ weeks GA (76.3\%) than those born at $28-31^{6}$ weeks GA (80.9\%). This may be because lower GA infants were more likely to be born by emergency delivery without adequate time for ACS administration. Our finding of an association between any administration of ACS and the rate of early death or severe brain injury is consistent with other reports in the literature [7] and reinforces the need for vigilance in administering ACS to mothers at risk of preterm delivery. Our finding that ACS was significantly $(p<0.05)$ associated with early death or severe brain injury among infants born at $24-27^{6}$ weeks GA (aOR 0.58 [95\% CI: $0.39,0.87]$ ) but not $28-31^{6}$ weeks GA (aOR 0.77 [95\% CI: 0.60, 1.00]) is consistent with Travers et al. [7]'s report that the benefits of ACS were larger among infants of lower GA.

Although $83.5 \%$ of infants in this cohort were inborn in tertiary hospitals, only $57.6 \%$ of them were normothermic at NICU admission, suggesting that measures to prevent hypothermia are inadequate. Rodrigo et al. [17] reported that hypothermia is associated with an increased risk of severe intraventricular hemorrhage and mortality. Strategies that have been shown to be successful include the use of plastic bags, wraps, caps, exothermic heat, and mattresses and can be easily adopted in the delivery room and NICU. Of note, in this study, NT was defined as 36.0$37.5^{\circ} \mathrm{C}$ measured at the first recorded temperature on NICU admission. The lower limit was $36^{\circ} \mathrm{C}[18]$, not the $36.5^{\circ} \mathrm{C}$ recommended by the WHO. This may lead to an overestimation of the proportion of NT used compared to other studies.

Brookfield et al. [19] reported that the use of prenatal $\mathrm{MgSO}_{4}$ for fetal neuroprotection before 32 weeks of pregnancy at standard dose protocol improved neonatal neurological outcomes, but there is currently no evidence that it reduces neonatal mortality. We did not find a lower rate of severe brain injury with administration of $\mathrm{MgSO}_{4}$, which is consistent with the report of Doyle et al. [8]. In this study, since $\mathrm{MgSO}_{4}$ was defined as the use of magnesium sulfate before delivery for any reason, and not only neuroprotection, it is possible that this may have diluted the results. However, we also found that use of $\mathrm{MgSO}_{4}$ in combination with ACS resulted in a lower incidence of early death or severe brain injury than ACS alone, which is consistent with the report of Gentle et al. [20] that the neuroprotective effect of $\mathrm{MgSO}_{4}$ is enhanced when used in combination with antenatal corticosteroids.
Jin et al. 
Since 2 of the 4 evidence-based practices in this study are maternal interventions and 2 are neonatal interventions, our study reinforces the need for close collaboration between obstetricians and neonatologists to optimize infant outcomes. Consistent with previous studies $[15,21]$, our study also shows that exposure to multiple evidence-based practices was associated with a lower risk of early death or severe brain injury than single exposures. Our finding that exposure to 3 practices was just as effective as 4 practices suggests that population or clinical practice differences may lead to real-world variations in efficacy.

\section{Limitations of This Study}

Our study had some limitations. Data were unavailable for other evidence-based practices such as use of surfactant within $2 \mathrm{~h}$ of birth and early nasal continuous positive airway pressure [22], deferred cord clamping [23], and umbilical cord milking [24]. There were 960 cases with missing information on one of the 4 practices and 965 cases with missing information on the outcome. We did not have information on long-term neurodevelopmental outcomes. We did not examine other perinatal interventions, including those occurring before NICU admission, that may impact outcomes.

\section{Conclusion}

Our research shows that the application of evidencebased healthcare practices can improve survival without severe brain injury in very preterm infants who are born at $24-31^{6}$ weeks GA. Exposure to multiple evidencebased practices was associated with a lower incidence of early death or severe brain injury. Close collaboration between obstetrics and neonatology is critical to improving outcomes.

\section{Acknowledgment}

See online supplementary material.

\section{Statement of Ethics}

This study was approved by the Ethics Review Board of Children's Hospital of Fudan University, Approval No. 2018-296. Waiver of consent was granted at all sites.

\section{Conflict of Interest Statement}

The authors have no conflicts of interest to declare.

\section{Funding Sources}

This study is supported by the Canadian Institutes of Health Research (CTP87518) and the Chinese Neonatal Network coordinating center.

\section{Author Contributions}

Hui Wu supervised the study; conceptualized and designed the study; and acquired, analyzed, and interpreted the data. Shoo K. Lee and Yun Cao supervised the study. Di Jin conceptualized and designed the study; drafted the manuscript; and acquired, analyzed, and interpreted the data. Xinyue Gu, Siyuan Jiang, and Yanchen Wang acquired, analyzed, and interpreted the data. Tongling Yang and Yulan Lu administrated and provided technical and material support. All authors critically revised the manuscript for important intellectual content, approved the final manuscript as submitted, and agree to be accountable for all aspects of the work.

\section{Data Availability Statement}

All data generated or analyzed during this study are included in this article. Further enquiries can be directed to the corresponding author.

\section{References}

1 Chawanpaiboon S, Vogel JP, Moller AB, Lumbiganon P, Petzold M, Hogan D, et al. Global, regional, and national estimates of levels of preterm birth in 2014: a systematic review and modelling analysis. Lancet Glob Health. 2019 Jan;7(1):e37-46.

2 Godeluck A, Gérardin P, Lenclume V, Mussard C, Robillard PY, Sampériz S, et al. Mortality and severe morbidity of very pre- term infants: comparison of two French cohort studies. BMC Pediatr. 2019 Oct;19(1): 360.

3 World Health Organization (WHO). WHO recommendations on interventions to improve preterm birth outcomes. Geneva: WHO; 2015.

4 Sasaki Y, Ishikawa K, Yokoi A, Ikeda T, Sengoku K, Kusuda S, et al. Short- and long-term outcomes of extremely preterm infants in Japan according to outborn/inborn birth status. Pediatr Crit Care Med. 2019 Oct;20(10):9639.

5 Lasswell SM, Barfield WD, Rochat RW, Blackmon L. Perinatal regionalization for very low-birth-weight and very preterm infants: a meta-analysis. JAMA. 2010 Sep; 304(9):992-1000. 
6 Carlo WA, McDonald SA, Fanaroff AA, Vohr BR, Stoll BJ, Ehrenkranz RA, et al. Association of antenatal corticosteroids with mortality and neurodevelopmental outcomes among infants born at 22 to 25 weeks' gestation. JAMA. 2011 Dec;306(21):2348-58.

7 Travers CP, Clark RH, Spitzer AR, Das A, Garite TJ, Carlo WA. Exposure to any antenatal corticosteroids and outcomes in preterm infants by gestational age: prospective cohort study. BMJ. 2017 Mar;356:11039.

8 Doyle LW, Crowther CA, Middleton P, Marret S, Rouse D. Magnesium sulphate for women at risk of preterm birth for neuroprotection of the fetus. Cochrane Database Syst Rev. 2009(1):CD004661.

9 Crowther CA, Middleton PF, Voysey M, Askie L, Duley L, Pryde PG, et al. Assessing the neuroprotective benefits for babies of antenatal magnesium sulphate: an individual participant data meta-analysis. PLoS Med. 2017 Oct;14(10):e1002398.

10 Lyu Y, Shah PS, Ye XY, Warre R, Piedboeuf B, Deshpandey A, et al. Association between admission temperature and mortality and major morbidity in preterm infants born at fewer than 33 weeks' gestation. JAMA Pediatr. 2015 Apr;169(4):e150277.

11 Cao Y, Jiang S, Sun J, Hei M, Wang L, Zhang $\mathrm{H}$, et al. Assessment of neonatal intensive care unit practices, morbidity, and mortality among very preterm infants in China. JAMA Netw Open. 2021 Aug;4(8):e2118904.
12 Richter L, Ting J, Muraca G, Synnes A, Lim K, Lisonkova S. Temporal trends in neonatal mortality and morbidity following spontaneous and clinician-initiated preterm birth in Washington State, USA: a population-based study. BMJ open. 2019 Feb;9(1):e023004.

13 Kong X, Xu F, Wu R, Wu H, Ju R, Zhao X, et al. Neonatal mortality and morbidity among infants between 24 to 31 complete weeks: a multicenter survey in China from 2013 to 2014. BMC pediatrics. 2016 Nov; 16(1):174.

14 Canadian Neonatal Network. CNN 2019 annual report. 2019. Available from: http:// www.canadianneonatalnetwork.org/portal.

15 Zeitlin J, Manktelow BN, Piedvache A, Cuttini M, Boyle E, van Heijst A, et al. Use of evidence based practices to improve survival without severe morbidity for very preterm infants: results from the EPICE population based cohort. BMJ. 2016 Jul;354:i2976.

16 Chow SSW, Creighton P, Chambers GM, Lui K. Report of the Australian and New Zealand neonatal network 2017. Sydney: ANZNN; 2019.

17 García-Muñoz Rodrigo F, Rivero Rodríguez S, Siles Quesada C. [Hypothermia risk factors in the very low weight newborn and associated morbidity and mortality in a neonatal care unit]. An Pediatr. 2014 Mar;80(3):14450.

18 Vermont Oxford Network. Vermont Oxford Network database: 2007 annual NICU quality management report. Burlington, VT: Vermont Oxford Network; 2008.
19 Brookfield KF, Vinson A. Magnesium sulfate use for fetal neuroprotection. Curr Opin Obstet Gynecol. 2019 Apr;31(2):110-5.

20 Gentle SJ, Carlo WA, Tan S, Gargano M, Ambalavanan N, Chawla S, et al. Association of antenatal corticosteroids and magnesium sulfate therapy with neurodevelopmental outcome in extremely preterm children. Obstet Gynecol. 2020 Jun;135(6):1377-86.

21 Rizzolo A, Shah PS, Boucorian I, Lemyre B, Bertelle V, Pelausa E, et al. Cumulative effect of evidence-based practices on outcomes of preterm infants born at $<29$ weeks' gestational age. Am J Obstet Gynecol. 2020 Feb;222(2): 181.e1-e10.

22 Sweet DG, Carnielli V, Greisen G, Hallman M, Ozek E, Te Pas A, et al. European consensus guidelines on the management of respiratory distress syndrome: 2019 update. Neonatology. 2019;115(4):432-50.

23 Kc A, Rana N, Målqvist M, Jarawka Ranneberg L, Subedi K, Andersson O. Effects of delayed umbilical cord clamping vs early clamping on anemia in infants at 8 and 12 months: a randomized clinical trial. JAMA Pediatr. 2017 Mar;171(3):264-70.

24 Toledo JD, Rodilla S, Pérez-Iranzo A, Delgado A, Maazouzi Y, Vento M. Umbilical cord milking reduces the risk of intraventricular hemorrhage in preterm infants born before 32 weeks of gestation. J Perinatol. 2019 Apr; 39(4):547-53. 